\title{
Incorporación de simulaciones en el laboratorio de Química general: influencia en el dominio afectivo del aprendizaje
}

\section{Simulations in Chemistry laboratory: influence in the affective learning domain}

\author{
Raquel Fernández Cézar \\ Universidad de Suffolk, Campus de Madrid \\ Universidad de Castilla La Mancha
}

Resumen: El objetivo de esta acción didáctica es diseñar un curso de laboratorio de Química general universitario incluyendo experimentos manuales y simulaciones y determinar en los alumnos la influencia de dicho diseño. Con este nuevo diseño del curso de laboratorio se persigue conseguir una mejora cualitativa en el aprendizaje de los alumnos y detectar su percepción respecto al uso de simulaciones en el laboratorio. Se mide la influencia en el dominio afectivo mediante una encuesta y en el dominio cognitivo mediante una prueba de evaluación. Se detecta una mejora en el dominio afectivo del aprendizaje en general, y en los alumnos que están a priori menos motivados en particular, pero no se consigue determinar cuánto afecta al dominio cognitivo.

Palabras clave: diseño de curso, laboratorio de Química, simulaciones, alumnos universitarios, dominio afectivo del aprendizaje.

Abstract: The main purpose of this work is the design of a Chemistry Laboratory course by means of manual and computerized experiment to achieve an improvement in the learning of Chemistry in first year university students, and to detect their perception about this design. With the new designed course it is pursued the improvement of the learning goals of students and the collection of their perceptions. The students' perception is measured through a survey and the influence on their learning through an exam consisting on the performance of an experiment. It is detected an improvement in the affective domain of learning in general, and particularly in students with lower motivation, but is not quantified the influence in the cognitive domain.

Key Words: course design, Chemistry laboratory, simulations, university students, affective domain in learning.

(Fecha de recepción: junio, 2013, y de aceptación: septiembre, 2014)

DOI: 10.7203/DCES.28.2630 


\section{Introducción}

El trabajo que se presenta se centra en la modificación de la parte práctica del curso General Chemistry (Química General) de 4 créditos, conocida como Chemistry Laboratory o Chem111Lab (Laboratorio de Química General), que corresponde a 1 crédito de los 4 totales de la asignatura. Se cursa en Suffolk University, Madrid Campus, que es uno de los campus instalados en nuestro país en los que pueden cursarse estudios en el sistema educativo universitario estadounidense. Los alumnos de este campus no son solo de origen estadounidense o español, sino internacional, y tienen por lo tanto distintos niveles iniciales en la asignatura que nos ocupa al provenir de diversos sistemas educativos, ya que en algunos países los cursos de Química en la formación preuniversitaria son obligatorios y en otros no. En el sistema educativo estadounidense, sistema en el que está enmarcada esta universidad, tanto los alumnos de ciencias como los que no lo son tienen que tomar una asignatura obligatoria de ciencias con laboratorio. Nuestros alumnos son, por lo tanto, estudiantes de ciencias (de la salud o ingenierías) y/o de carreras no científicas.

La disminución de alumnos en las carreras científicas se viene produciendo en los últimos años en todas las universidades de los países occidentales, como recoge el estudio del Ministerio de Educación, Cultura y Deporte Español (2012) con referencias a las últimas dos décadas. Este campus que nos ocupa, a la escala que le corresponde, no escapa a esta evolución. Así, ha pasado en este curso de Química de tener solo estudiantes de ciencias en los años 90 y primeros 2000, a tener aproximadamente la mitad en los últimos años. Los resultados obtenidos por estos estudiantes, y la marcha diaria de las clases en esta nueva situación ha hecho que los docentes de este curso se hayan replanteado la idoneidad de la metodología docente empleada en la enseñanza de la Química, y sobre todo de la parte experimental, en la que los alumnos se sienten más incómodos y por la que muchos muestran bastante rechazo.

Para abordar la enseñanza de Química en grupos tan heterogéneos, los profesores optan por cambiar las metodologías con las que imparten sus clases. De esta forma, y como se recoge en la bibliografía, algunos profesores se decantan por el uso de tablillas de colores (clickers) en las clases teóricas. Cada color se corresponde con entendido o no entendido, y permite al profesor saber rápidamente si sigue adelante $o$ vuelve a incidir en el tema explicado. Parecen conseguir con ellos (Woelk, 2008) despertar o aumentar el interés en los alumnos en clases muy numerosas. Otros profesores optan por esa misma técnica añadiendo el trabajo cooperativo y las explicaciones entre iguales (Mazur, 1997). Y otros autores, como Kovac (1999), proponen el uso de los test conceptuales (concept test) para conseguir un aprendizaje activo por parte de los alumnos, también para grandes grupos y en las clases teóricas. 
Sin embargo, hay también casos en los que los profesores optan por actuar sobre las experiencias del laboratorio de Química con el fin de que estas resulten atractivas a los estudiantes (Bailey, Kingsbury, Kulinowski, Paradis, Schoonover, 2000) y les ayuden a adquirir los conocimientos y habilidades que se persiguen con el curso.

En nuestros grupos de alumnos el mayor rechazo surge en la parte experimental donde detectamos una escasa motivación, que creemos que redunda en un rendimiento más bajo del esperado, a juzgar por su trabajo y resultados académicos obtenidos en la clase teórica.

\section{Marco teórico y antecedentes}

Estudios previos acerca de la situación general en los laboratorios universitarios en España y en otros países occidentales (Tortosa y Gorchs, 2011), como EE.UU. (Bopegedera, 2011), muestran que en los primeros cursos universitarios es frecuente que los profesores que se encargan de impartir las asignaturas de prácticas no sean especialistas en la carrera docente (Teaching Assistant). De esta forma, la impartición de estas asignaturas es poco cuidada, y la ejecución de experiencias se realiza siguiendo guiones pautados con actividades cerradas (Domin, 1999).

Es también habitual que la evaluación del trabajo se base en informes escritos (Hofstein, 2004). Algunas veces se puede tener la sensación de que el alumnado sigue el protocolo de laboratorio como si de una receta se tratase, respondiendo a las cuestiones de los informes de una manera mecánica más que razonada. Este hecho queda reflejado en informes muy iguales por parte de alumnos que son muy diferentes, lo que hace dudar o de la autoría de los informes, o de la medida en que estos reflejan la comprensión de lo tratado.

Se han encontrado acciones sobre la parte experimental de los cursos de Química como la de Bopegedera (2011), que él llama LCA (Laboratory Centered Approach) o Metodología Centrada en el Laboratorio. En esta propuesta el instructor realiza la preparación de los guiones de laboratorio para cada práctica. Sin embargo no contempla la introducción de laboratorios virtuales a ejecutar por los alumnos.

Sobre el uso de laboratorios virtuales, experimentos reales, y la combinación de ambos existen muchos estudios realizados. La conclusión aceptada por la mayoría de investigadores es que se consigue un mejor aprendizaje con el uso de ambos combinados en diferentes proporciones a lo largo de los cursos: bien de manera secuencial realizando el mismo experimento de una y otra forma (Jaakkola y Nurmi, 2008; Jaakkola, Nurmi y Veermans, 2010), o bien utilizando experimentos reales o virtuales para distintos conceptos químicos (Gire, Carmichael, Chini, Rouinfar, Rebello y Smith, 2010; Zacharia y Constantinous, 2008; Georgios y Zacharia, 2012). En lo que no hay acuerdo unánime es en cuál es la combinación o proporción óptima en aras de conseguir un mejor aprendizaje de los conceptos 
por parte de los alumnos. Sí hay acuerdo, sin embargo, en que las habilidades y conocimientos que permite alcanzar a los estudiantes cada uno de los modelos de laboratorio son complementarios.

Las ventajas que en la bibliografía sobre este tema se le reconocen a los experimentos manualmente desarrollados (Balamuralithara y Woods, 2009; Hofstein y Lunetta, 2004) son principalmente tres: que permiten adquirir habilidades psicomotoras; que permiten ser conscientes de la necesidad de seguir los protocolos de seguridad; y que permiten el aprendizaje a través de la observación. Las que se le reconocen a los virtuales (de Jong y Njoo, 1992; Windschitl, 2000; Winn. Stahr, Sarason, Fruland, Oppenheimer y Lee, 2006) son variadas, y entre ellas se destacan las siguientes: permiten la simplificación de los modelos del mundo real, por lo que transforman los fenómenos en más visualizables para los estudiantes acomodándose a los distintos niveles individuales de adquisición de conocimientos; proporcionan mucha información y representaciones múltiples (verbal, numérica, conceptual, visual, gráfica) permitiendo aprender a alumnos con distintas inteligencias (Sternberg, Jarvin y Grigorenko, 2009); permiten repetir inmediatamente y cuantas veces sea necesario el experimento focalizando la atención de los estudiantes en el fenómeno a aprender. Nosotros pensamos que tienen otras dos ventajas que destacamos, y son, la primera que posibilitan que aquellos alumnos con escasas habilidades manuales puedan demos- trar otras habilidades de análisis, sin que se lo impida su carencia de las primeramente nombradas; y la segunda, que nos permite realizar experimentos en nuestro laboratorio de manera virtual que de manera real sería imposible llevarlos a cabo, bien por el coste económico del material, bien por las medidas de seguridad necesarias.

El análisis de la situación de partida en estos cursos de laboratorio y la posterior mejora de las prácticas es abordado mayoritariamente en la bibliografía de investigación e innovación educativa mediante tres acciones: una de ellas la constituyen las opiniones de profesorado y alumnado acerca de la materia y las posibilidades de mejora, otra es la mejora de los tipos de guiones utilizados y actividades de evaluación, y la tercera es el estudio de la gestión de las actividades de aula. En este trabajo desarrollamos la primera principalmente, y en cierta medida la tercera, si bien nuestros datos se refieren mayoritariamente a la primera.

Las hipótesis de investigación en nuestro trabajo son:

1. La combinación adecuada de experimentos manuales/virtuales en un curso de laboratorio de Química general debe ser tal que permita a los alumnos adquirir las habilidades psicomotoras y analíticas básicas y les posibilite una visión completa del trabajo científico.

2. Con la utilización de simulaciones en el curso Laboratorio de Química combinadas con las prácticas manuales mejoraremos la percep- 
ción de los alumnos de este curso, actuando positivamente sobre su dominio afectivo.

3. La mejora de su motivación conllevará una mejora de su rendimiento académico.

\section{Metodología}

Este trabajo se basa en el método de la investigación-acción: se realiza el análisis de la situación inicial por los docentes que son profesores cualificados y con experiencia en impartir esos laboratorios, y la acción es dirigida por ellos puesto que a la vez son los investigadores. Por ello van proponiendo cambios a lo largo de los cursos tras el consiguiente proceso de reflexión. Estos cambios se llevan a cabo en el aula y se analiza la nueva situación mediante encuestas en las que se recoge la percepción de los alumnos. Se pretende seguir repitiendo el ciclo de revisiones hasta conseguir una situación óptima.

El objetivo del trabajo es dual y consiste, por un lado en encontrar un diseño adecuado del curso de laboratorio que combine experimentos manuales y virtuales para que nuestros alumnos adquieran las habilidades básicas esperadas en un estudiante de un curso de Química general, y por otro, que esto contribuya a mejorar su motivación en estas prácticas de Química, y posiblemente, su rendimiento.

La muestra está compuesta por 54 alumnos que son los que han pasado por este curso de laboratorio en el período 2008-20013. Se analiza la composición de esta muestra atendiendo al porcentaje de alumnos de carreras de ciencias, ya sean de la salud o ingenierías, así como la distribución de las notas en el laboratorio de Química.

El grupo de alumnos que no tienen simulaciones en el curso de laboratorio constituye nuestro grupo "control" (GC) y corresponde a los años 2008 y 2009 (24 alumnos) y el grupo que llamaremos "experimental" (GE) lo constituyen los alumnos que ya han tomado el curso con la nueva combinación de experimentos manuales/virtuales, y corresponden a los años 2010 a 2013 (30 alumnos).

El diseño del curso y combinación de experimentos manuales y virtuales se determina en función de las habilidades que el syllabus del curso recoge para nuestros alumnos, que son: manejo y elección de aparatos y utensilios adecuados para realizar medidas de masa, medidas de volumen, preparación de disoluciones, diluciones, titulaciones, cálculos de masa y volumétricos de concentraciones, así como calor de reacción. Por ello se decide que realicen de manera manual aquellos experimentos con los que los estudiantes adquieren las habilidades psicomotoras básicas de las nombradas anteriormente, y mediante simulaciones aquellas que no añaden más habilidades manuales y sin embargo les permiten centrarse en el análisis cuantitativo posterior de los datos.

Para elegir las simulaciones a emplear, se han realizado búsquedas en internet. De las encontradas hemos seleccionado dos páginas web por razones pedagógicas: animationindex, de 
Iowa State University, y la página de simulaciones interactivas del grupo PhET de investigación en didáctica de ciencias en University of Colorado at Boulder. Finalmente nos hemos quedado con la primera página puesto que las simulaciones que aparecen en la segunda no nos han resultado útiles para implementar los laboratorios que incluimos en este curso.

La valoración de la percepción del laboratorio se mide mediante una encuesta preparada para la ocasión, que se pasa a los alumnos del grupo experimental. La percepción del grupo de control no se recoge con esta encuesta, pero los comentarios que recibíamos al final de cada curso son los que desencadenaron el cambio de diseño del curso que se muestra en este trabajo. Se analizan las respuestas en relación a su percepción de la inclusión de simulaciones en el curso y a la influencia que ellos esperan que estas tengan en su aprendizaje.

En la bibliografía encontramos que las encuestas son uno de los métodos frecuentemente utilizados para tener información sobre las opiniones tanto de profesores como de alumnos (Insausti, 1997; Cheung, 2008). Respecto a la gestión del aula, las actividades que llevan a cabo los estudiantes se muestran determinantes en la mejora de su rendimiento en el laboratorio (Tortosa y Gorchs, 2006). También encontramos que influyen positivamente en los resultados de aprendizaje las actividades previas a las prácticas que realizan los estudiantes, y el aprendizaje cooperativo (Domingo, 2003; Barbosa et al, 2004).
Teniendo esto en cuenta, para despertar el interés de los alumnos empleamos guiones que van acompañados de unas preguntas previas al desarrollo de la práctica (prelab questions) y otras a contestar al final de la misma para guiarles hacia las conclusiones (post lab questions). Así mismo, los laboratorios se realizan en parejas o grupos de tres como máximo, facilitando la interacción y colaboración entre los estudiantes y entre ellos y el profesor.

Finalmente, se comparan los resultados académicos de los alumnos en esta asignatura del grupo control y experimental, para determinar, si existiera, una mejora perceptible en los resultados de los mismos.

\section{Resultados}

\section{Diseño del curso}

El curso de laboratorio de Química que se emplea está compuesto por 8 experimentos de los que se realizan los primeros 6 de manera manual, y los 2 últimos mediante simulaciones. Esta combinación nos parece la más adecuada porque combina actividades que permiten aprender a estudiantes con distintos tipos de habilidades e inteligencias: habilidades psicomotoras mediante los experimentos 1-6, habilidades visuales y analíticas en los experimentos 7 y 8 . Esto no va en detrimento de la formación de los estudiantes ya que las habilidades psicomotoras que se usarían en estos experimentos si se realizaran de forma manual ya han sido adquiridas 
con los experimentos 1-6, y así el estudiante puede poner el énfasis en los análisis cuantitativos a realizar, como el cálculo del calor de neutralización, el calor de combustión y el significado de reactivo limitante en las reacciones. De esta forma dividimos el aprendizaje en dos grandes estadios: adquisición de habilidades psicomotoras y adquisición de habilidades de análisis cuantitativo y estequiométrico de reacciones. Con nuestro planteamiento de curso, el hecho de no adquirir las primeras no impide a los estudiantes la adquisición de las segundas.

Las prácticas que componen el laboratorio son las siguientes:

1. Mass measurements. (Medidas de masa)

Descripción: Se muestra la diferencia entre masa y peso y se pide obtener la masa de reactivos sólidos, en polvo y líquidos en balanzas con distinta precisión: digital y analítica. Se obtienen las masas de los reactivos mediante pesada del contenedor y conjunta, y mediante la función tara de la balanza, para observar la diferencia.

2. Calibration of Glassware. (Calibración de aparatos de vidrio)

Descripción: Se calibran distintos utensilios para medir volumen (pipeta y matraz aforado de $100 \mathrm{~mL}$ ) mediante pesada de distintos volúmenes medidos con estos utensilios. Se aplica el test $\mathrm{Q}$ a los resultados para determinar la precisión del calibrado.
3. Preparation of solid reagent solutions-dilutions.(Preparación de disoluciones de soluto sólido- diluciones)

Descripción: Se emplean las técnicas aprendidas en los dos laboratorios anteriores para obtener una masa determinada de un reactivo sólido en polvo y el volumen necesario para preparar una disolución de concentración (molaridad) dada. También se procede a realizar una dilución desde disolución comercial a una concentración pedida. En ambos casos se realizan primero los cálculos necesarios para determinar la cantidad de soluto sólido y de reacción más concentrada, respectivamente.

4. Observation and interpretation of chemical Change. (Observación e interpretación del cambio químico) Descripción: Se analizan cualitativamente distintos tipos de reacciones utilizando la observación y prediciendo/comprobando lo que ocurre mediante la ecuación iónica neta.

5. pH measurements. (Medidas de $\mathrm{pH}$ ) Descripción: Se emplea un $\mathrm{pH}$ metro de campo para tomar medidas del $\mathrm{pH}$ de distintas sustancias, recalcando que deben estar en disolución para poder medirlo, y se calcula también la concentración de iones $\mathrm{H}+$ en disolución.

6. Acid base titration. (Titulaciones ácido-base) 
Descripción: Se titula biftalato de potasio, un ácido débil, de concentración desconocida, con sosa $1 \mathrm{M}$. Se emplea como indicador la fenolftaleína. Se repite el procedimiento varias veces y se toma la media y se calcula la desviación típica.

7. Acid-base reactions: Heat of neutralization. (Reacciones ácidobase: calor de neutralización)

Descripción: ver anexo 1.

8. Heat of combustión. (Calor de combustión)

Descripción: ver anexo 2.

La práctica 7 se encuentra en la página del Chemical Education Research Group, Department of Chemistry, Iowa State University (EE.UU.) en la sección de Thermochemistry (TermoQuímica) con título Heat of neutralization experiment version 2 (Greenbowe, R.), y para ejecutar el experimento 8 se elige la simulación que se encuentra en esa misma página web en el apartado de Stoichiometry (Estequiometria) y que se llama Combustion reactions of hydrocarbons with oxygen gas and limiting reagent (Reacciones de combustión de hidrocarburos con oxígeno gas y reactivo limitante). La última de las elegidas, la número 8 , simula un experimento que no sería posible llevar a cabo en nuestras instalaciones de manera manual.

Estas simulaciones son de uso libre y pueden descargarse o usarse en línea. Esta página web ha sido elaborada por el grupo del profesor Greenbowe, y en ella se encuentra no solo esta simulación, sino muchas otras relativas a diversos temas de Química introductoria, desde estequiometria a reacciones redox y electroQuímica, pasando por las leyes de los gases, termoQuímica, reacciones ácido-base y equilibrio químico.

Entendemos que esta distribución es adecuada porque contribuye a que los alumnos con distintos tipos de inteligencias tengan variadas oportunidades de aprendizaje y que la separación entre habilidades manuales y de cálculo es positiva para la diversidad del estudiantado.

\section{Valoración de la inclusión de} simulaciones y resultados académicos

Contamos con el registro de alumnos en los últimos años en la asignatura que nos ocupa y se realiza un análisis de los mismos, así como de las carreras elegidas por ellos (major, que equivaldría a la licenciatura/grado en nuestro sistema), con el fin de saber si la que cursa el alumno es de ámbito científico o no. Al ser esta una asignatura que se elige en su primer curso en la universidad, Freshman year, algunos alumnos no tienen claro aún qué van a hacer, por lo que es pertinente la inclusión de un apartado "No sabe". Las categorías elaboradas son, por lo tanto, Ciencias, No ciencias, y No sabe, y el porcentaje de alumnos en cada una se muestra en la figura I.

Se elabora la encuesta compuesta por 7 preguntas que se responden en una escala de 1 a 5 , desde totalmente de acuerdo (TA) valorado con 1 , hasta totalmente en desacuerdo (TD) valorado con 5 , pasando por ni de acuerdo ni 
Figura I: Porcentaje promedio de alumnos en los cursos 2008-2013 que cursan carreras de Ciencias, No ciencias y No sabe

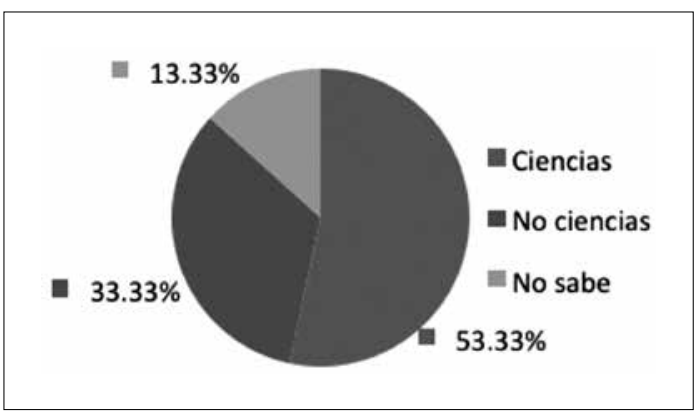

en desacuerdo, valorado con 3. Se elige este tipo de respuesta porque es el más adecuado a la mayoría de las preguntas. Estas se formulan de forma afirmativa unas y negativa otras, de manera que no siempre TA es la respuesta más positiva, o TD la más negativa. Pretendemos así evitar que aquellos estudiantes que marquen en todas el mismo número sin leer la pregunta puedan contribuir a darnos mejores o peores resultados medios. Cada pregunta tiene también un apartado de comentarios o sugerencias, dejándola así abierta a la reflexión de los estudiantes sobre lo que se les pregunta. Se pasa a los alumnos del grupo experimental, que ascienden a 30 . Dado que el $46,66 \%$ de los alumnos no creen que vayan a realizar una carrera científica (figura I), eso supone que, a priori, sean los estudiantes menos motivados para las clases de laboratorio, que las sesiones que lo componen no les resulten muy atractivas $\mathrm{y}$ que sea necesario motivarles en mayor medida que a aquellos que eligen carreras de ciencias, que tendrán más disposición a realizar este tipo de trabajo. Sin embargo no todos los estudiantes de ciencias muestran habilidades psicomotoras o gusto por adquirirlas, ni son amantes de las prácticas de laboratorio al estilo tradicional. Antes al contrario defienden la tecnología y dado que su uso es generalizado en los laboratorios reales esperan un planteamiento de los laboratorios en los cursos básicos de formación que la incluyan.

La encuesta completa se incluye en el anexo 3. No todos los estudiantes han contestado todas las preguntas y aquellas en las que solo había una o dos preguntas contestadas no se han considerado. Los resultados obtenidos se detallan en la tabla siguiente (Tabla I)

Puesto que en la encuesta se asigna 1 a totalmente de acuerdo (TA) y 5 a totalmente en desacuerdo (TD), los valores medios bajos muestran percepción más positiva por parte de los alumnos, dada la formulación de las preguntas. Los valores medios de todas las preguntas están cercanos a 2 , lo que muestra que los estudiantes están de acuerdo con lo que les propone la pregunta.

A juzgar por esos datos, podemos decir que están de acuerdo en la pregunta 1 , y consideran que las simulaciones les han ayudado; también están de acuerdo en que son más útiles que los ejercicios numéricos (pregunta 3), en que han entendido las reacciones ácidobase cualitativamente (pregunta 4), en que han entendido las reacciones ácidobase cuantitativamente (pregunta 5), 
Tabla I: Percepción de los estudiantes del grupo experimental sobre los laboratorios realizados con simulaciones, $\mathbf{n}=30$. Incluye los valores medios de las respuestas del estudiantado calculados asignando valores entre 1 y 5 a respuestas entre TA y TD para todas las preguntas.

\begin{tabular}{|c|c|c|c|c|c|c|}
\hline $\begin{array}{l}\text { Encuesta sobre empleo de } \\
\text { simulaciones en el } \\
\text { laboratorio de Química } \\
\text { General }\end{array}$ & $\mathbf{n}$ & Máximo & Mínimo & $\begin{array}{l}\text { Valoración } \\
\text { media }\end{array}$ & $\begin{array}{l}\text { Desviación } \\
\text { típica }\end{array}$ & Rango \\
\hline $\begin{array}{l}\text { P.1: Las simulaciones me han } \\
\text { ayudado. }\end{array}$ & 30 & 5 & 1 & 2,06 & 0,88 & 4 \\
\hline $\begin{array}{l}\text { P.2: Te han ayudado a entender las } \\
\text { reacciones involucradas. }\end{array}$ & 29 & 4 & 1 & 2,77 & 1,02 & 3 \\
\hline $\begin{array}{l}\text { P.3: Son más útiles que los } \\
\text { ejercicios numéricos. }\end{array}$ & 29 & 5 & 1 & 2,03 & 0,95 & 4 \\
\hline $\begin{array}{l}\text { P.4: Has entendido las reacciones } \\
\text { ácido-base cualitativamente. }\end{array}$ & 29 & 4 & 1 & 2,13 & 0,92 & 3 \\
\hline $\begin{array}{l}\text { P.5: Has entendido las reacciones } \\
\text { ácido-base cuantitativamente. }\end{array}$ & 29 & 4 & 1 & 2,23 & 1,02 & 3 \\
\hline $\begin{array}{l}\text { P.6: Has entendido cualitativamente } \\
\text { las reacciones de combustión. }\end{array}$ & 29 & 4 & 1 & 2,14 & 1,03 & 3 \\
\hline $\begin{array}{l}\text { P.7: Has entendido cómo se trata } \\
\text { el flujo del calor en las reacciones } \\
\text { de combustión. }\end{array}$ & 29 & 5 & 1 & 2,00 & 1,09 & 4 \\
\hline
\end{tabular}

en que han entendido las reacciones de combustión (pregunta 6), así como cómo tratar el flujo de calor en estos tipos de reacciones (pregunta 7 ).

La segunda pregunta es la única que presenta un valor medio más cercano a 3 , ni de acuerdo ni en desacuerdo, por lo que es en la que los estudiantes muestran una actitud más neutra. Es verdad que es neutro el valor medio en esta pregunta, pero los resultados muestran la variedad de percepción de los alumnos ya que hay algunos que han contestado en desacuerdo (8) y otros totalmente de acuerdo (5). Eso nos hace ver que hay disparidad de opiniones, y que puede encontrarse una aclaración a este punto con las observaciones y comentarios que también se incluyen en cada pregunta (ver tabla III). 
En cuanto a la desviación típica, indicador de la dispersión de los datos, la pregunta que más uniforme muestra la respuesta en torno al valor medio es la primera, es decir, que los estudiantes están de acuerdo en que las simulaciones les han resultado útiles para entender los conceptos que trataban. Sin embargo, el rango, medida estadística que se calcula como diferencia entre el valor mayor y el menor de una colección de datos dada, es máximo también en esta pregunta, lo que quiere decir que hay alumnos que las consideran de mucha ayuda, y también otros que no las consideran de ayuda en absoluto.

En la tabla II se muestran los porcentajes de respuesta por pregunta descartando las contestaciones NA/ND, agrupando TA-A, y D-TD.

\section{Tabla II: Agrupación de las respuestas de la tabla I en categorías TA-A y D-TD en porcentajes}

\begin{tabular}{|c|c|c|}
\hline Número de pregunta & \% respuestas entre TA y A & \% de respuestas entre D y TD \\
\hline P.1 & 59,4 & 9,4 \\
\hline P.2 & 22,6 & 61,3 \\
\hline P.3 & 58,1 & 29,0 \\
\hline P.4 & 66,7 & 20,0 \\
\hline P.5 & 25,0 & 52,0 \\
\hline P.6 & 54,8 & 25,8 \\
\hline P.7 & 54,8 & 32,2 \\
\hline
\end{tabular}

Con estos datos vemos que en las P.1, P.3, P.4, los porcentajes de respuesta entre TA y A están cerca del $60 \%$ e incluso superior; en las P.6 y P.7 el porcentaje es algo inferior pero aun así superior a la mitad e igual en ambas preguntas a $54.8 \%$. En las preguntas P.2 y P.5 más del $50 \%$ de las respuestas caen en las dos categorías D y TD.

Entendemos que este análisis cuantitativo mediante encuestas, a pesar de incluir el apartado de sugerencias donde permite a los estudiantes su aporte original, tiene un valor limitado, y que puede completarse con entrevistas a los alumnos. Pero en un primer nivel de análisis nos permite concluir que la percepción que los estudiantes tienen de esta estructura de curso de laboratorio incluyendo estas simulaciones es fundamentalmente positiva y contribuye a aumentar su motivación respecto a la 
asignatura de laboratorio de Química. Ellos consideran que les ayuda a aprender, que es, por otro lado, el objetivo perseguido por los profesores del curso.

En cada pregunta se incluía un apartado de comentarios/sugerencias, lo que ha permitido a los estudiantes aclarar por qué daban cada una de sus respuestas, o añadir otras cosas que ellos pensaban que eran pertinentes. De estos comentarios/sugerencias, se muestran los más representativos en la tabla III, separados en apartados de consideraciones positivas, negativas, y neutras, y algún otro comentario que hacen pero que no es pertinente considerar en este estudio (N/A).

Parte de estos comentarios se encuentran recogidos en la bibliografía sobre el tema de la que se habla en la introducción (deJong y Njoo, 1992; Windschitl, 2000; Winn y col, 2006), por lo que son generales y trascienden a nuestro grupo en particular. Son, por lo tanto, bastante universales e independientes del sistema y contexto educativo del que venga el estudiante.

Para determinar la influencia sobre el rendimiento académico, se comparan los resultados en la prueba final

\section{Tabla III. Comentarios y sugerencias aportadas por los alumnos del grupo experimental}

\begin{tabular}{|l|c|}
\hline Positivas: & Frecuencia \\
\hline Entiendo mejor las reacciones después de usar la simulación. & 3 \\
Permiten visualizar los procesos que tienen lugar en las reacciones. & 1 \\
Me permiten entender por qué hacemos algunos cálculos en problemas. & 3 \\
Son de mucha ayuda y aprehendo mejor los conceptos. & 5 \\
Simulaciones son útiles cuando te enfrentas a un concepto por primera vez. & 1 \\
Son más entretenidas que los experimentos reales. & 1 \\
Son más exactas porque no interviene el error humano. & 3 \\
\hline Neutras: & \\
Me gustan las simulaciones en las clases teóricas pero no en lugar de los & \\
laboratorios reales. & 1 \\
\hline Negativas: & 2 \\
No las entiendo al principio. & 1 \\
No son de ayuda para estudiantes que no tienen habilidad visual. & 3 \\
Nunca son tan útiles como los experimentos reales. & \\
Los experimentos reales ponen en juego muchos más sentidos que las & 1 \\
simulaciones & \\
\hline N/A: & 1 \\
\hline El curso es muy complicado para ser un curso introductorio de Química & \\
\hline
\end{tabular}


de laboratorio. Esta prueba consiste en la realización de una titulación ácidobase, ambos fuertes, empleando fenolftaleína como indicador. Incluye el cálculo previo del volumen necesario de la base para neutralizar el volumen dado del ácido. Esta práctica involucra tanto la muestra de las habilidades psicomotoras en el manejo del material típico de laboratorio (medidas de masa y volumen y preparación de disoluciones) como las habilidades de razonamiento y cálculo previo. Se pide hacer varias repeticiones y obtener el valor medio y desviación típica con calculadora o utilizando una hoja de cálculo.

En esta prueba se daba la opción de poder desarrollar el experimento con la simulación con la que se realiza el experimento 7. Ningún estudiante ha hecho uso de la simulación en la evaluación; sin embargo sí la han usado para com- probar los resultados de sus cálculos de la concentración desconocida.

Los resultados de la prueba de evaluación se muestran en la tabla IV englobando en el primer grupo a los estudiantes del grupo control, y en el segundo a los estudiantes de los cursos que constituyen el grupo experimental.

Al observar esta tabla, vemos que la información que aportan los valores medios es que para los alumnos de ciencias ese valor pasa de 8.3 a 8.7 , para los estudiantes de No ciencias sube ligeramente de 6.5 a 7.3 y para los de la categoría No sabe pasa de 6.5 a 6.8 . $\mathrm{Al}$ observar la columna del Rango, se observa una tendencia general: el rango se hace más estrecho desde el primero en todas las categorías de estudiantes. Este valor pasa de 3.5 a 2.7 en la categoría Ciencias; de 3.0 a 2.1 en la categoría No ciencias; queda prácticamente igual en la categoría No sabe. Al ser la

Tabla IV: Comparación de resultados mínimos, máximos y medios de los estudiantes de General Chemistry Laboratory entre el grupo control (GC) y el grupo experimental (GE). Las notas son sobre 10. Se incluye el rango como medida de dispersión

\begin{tabular}{|l|c|c|c|c|}
\hline Cursos 2008-2010 (GC) & Mínimo & Máximo & Media & Rango \\
\hline Ciencias & 6.0 & 9.5 & 8.3 & 3.5 \\
No ciencias & 4.5 & 7.5 & 6.5 & 3.0 \\
No sabe & 5.0 & 7.5 & 6.5 & 2.5 \\
\hline Cursos 2010-2013 (GE) & & & & \\
\hline Ciencias & 6.8 & 9.5 & 8.7 & 2.7 \\
No ciencias & 5.9 & 8.0 & 7.3 & 2.1 \\
No sabe & 5.0 & 7.7 & 6.8 & 2.7 \\
\hline
\end{tabular}


evolución de los rangos decreciente concluimos que los alumnos obtienen en la prueba resultados más uniformes.

Dado que el grupo control constituye una muestra pequeña y el experimental una muestra media, realizamos un test de igualdad o diferencia de los valores medios usando la t de student. Así encontramos que la media del grupo control es 7.5 con una desviación estándar de 1.9, y para el grupo experimental la media es 8.0 y la desviación estándar 1.5. Con estos valores podemos asegurar con el $75 \%$ de confianza que las medias son diferentes. Esto quiere decir que tenemos un nivel de confianza bajo para afirmar que la mejora detectada en la motivación de los estudiantes del grupo experimental se traduzca en una mejora del rendimiento académico medido con esta prueba de evaluación.

Se pretende seguir realizando el mismo tipo de observaciones en próximos cursos para comprobar la reproducibilidad de esta mejora y determinar si hay otras variables didácticas a modificar en el curso de laboratorio de Química que pudieran contribuir positivamente al aprendizaje integral de los estudiantes.

\section{Conclusiones}

De todo lo expuesto podemos decir que se prueban algunas de las hipótesis de partida, pero no todas. Se muestran a continuación las conclusiones:

Se ha diseñado un curso de Laboratorio de Química con 8 prácticas y con una combinación de prácticas manuales/virtuales de 6 a 2. Con las prácticas 1-6 el alumno desarrolla y adquiere las habilidades psicomotoras y manuales que se piden en el curso y con las prácticas 7 y 8 (simulaciones) las habilidades analíticas y cuantitativas que se le piden. Se propone el uso de la página web animationindex para el desarrollo de cualquier laboratorio virtual de Química introductoria por sus características de gratuidad y riqueza de contenidos recogidos.

Se ha valorado cómo perciben los alumnos este diseño de curso, en concreto la inclusión de las simulaciones, con una encuesta y se han obtenido resultados que muestran que esa percepción es muy positiva y que ha influido positivamente en su visión de este curso de laboratorio.

Con esta acción didáctica se ha conseguido actuar sobre el dominio afectivo del aprendizaje de los alumnos, pero no encontramos en nuestros datos una relación clara entre la mejora de la motivación y su mejora en el rendimiento académico, medido con la prueba de evaluación que se recoge en este trabajo.

El reto permanente de los docentes está en ofrecer a los alumnos de laboratorio de Química unos cursos cuyo diseño les traslade una visión más completa y actual del trabajo en un laboratorio, que les resulte motivador y que también les haga mejorar sus conocimientos. Para posteriores trabajos nos queda determinar con un mayor nivel de confianza si esto último lo conseguimos. 


\section{Referencias}

BAILEY, C. A., KINGSBURY, K., KULINOWSKI, K., PARADIS, J., SCHOONOVER, R. (2000), An integrated lecture-laboratory environment for General Chemistry, Journal ofChemical Education, 77(2), 195-199. DOI: 10.1021/ed077p195.

BALAMURALITHARA, B., Y WOODS, P. C. (2009), Virtual laboratories in engineering education: The simulation lab and remote lab. Computer Applications in Engineering Education, 17 (1), 108118. DOI: 10.1002/cae.20186.

BARBOSA, R., JOFILI, Z. Y WATTS, M. (2004), Cooperating in constructing knowledge:casestudiesfromchemistry and citizenship, International Journal of Science Education, 26 (8), 935-949. DOI: 10.1080/0950069032000138842.

BOPEGEDERA, A.M.R.P. (2011), Putting the laboratory at the center of teaching Chemistry, Journal of Chemical Education, 88 (4), 443-448. DOI: 10.1021/ed100045z.

CHEUNG, D. (2008), Facilitating Chemistry teachers to implement inquiry-based laboratory work, International Journal of Science and Mathematics Education, 6 (1), 107 130. DOI: $10.1007 / \mathrm{s} 10763-007-9102-y$.

DE JONG, T., Y NJOO, M. (1992), Learning and instruction with computer simulation: Learning processes involved, en E. DE CORTE, M.C. LINN, H. MANDL Y L. VERSCHAFFEL (eds.), Computer- based learning environments and problem solving (411-427). Berlin: Springer-Verlag.

DOMIN, D.S. (1999), A review of laboratory instruction styles, Journal of Chemical Education, 76 (4), 543547. DOI: 10.1021/ed076p543.

DOMINGO, J. (2003), El aprendizaje Cooperativo, una vía hacia la excelencia docente. En Comunicación presentada en III Congreso Internacional sobre Docencia Universitaria e Innovación, Gerona.

GIRE, E., CARMICHAEL, A., CHINI, J. J., ROUINFAR, A., REBELLO, S., Y SMITH, G. (2010, Junio), The effects of physical and virtual manipulatives on students' conceptual learning about pulleys, en Proceedings of the 9th international conference of the learning sciences, Vol. 1, (937-944). International Society of the Learning Sciences.

GEORGIOS O. Y ZACHARIAS C. Z. (2012), Blending physical and virtual manipulatives: An effort to improve students' conceptual understanding through science laboratory experimentation, Science Education, 96 (1), 21-47. DOI: 10.1002/sce.20463.

GREENBOWE R. RESEARCH GROUP, http://group.chem.iastate.edu/ Greenbowe/tg-research.html [2 de Julio de 2014]

HOFSTEIN, A. (2004), The laboratory in Chemistry Education: thirty years of experience with developments, implementation and research. 
Chemical Education Research and Practice, 5 (3), 247-264. DOI: 10.1039/ B4RP90027H

HOFSTEIN, A. Y LUNETTA, V. (2004), The laboratory in science education: Foundations for the twenty-first century, Science Education, 88 (1), 28-54. DOI: 10.1002/sce.10106

INTERACTIVE SIMULATIONS, http:// phet.colorado.edu/en/simulations/ index [2 de julio de 2014]

INSAUSTI, J.M. (1997), Análisis de los trabajos prácticos de Química general en un primer curso de universidad, Enseñanza de las Ciencias, 15 (1), 123-130.

JAAKKOLA, T., Y NURMI, S. (2008), Fostering elementary school students' understanding of simple electricity by combining simulation and laboratory activities, Journal of Computer Assisted Learning, 24 (4), 271 - 283. DOI: 10.1111/j.13652729.2007.00259.x.

JAAKKOLA, T., NURMI, S., Y VEERMANS, K. (2010), A comparison of students' conceptual understanding of electric circuits in simulation only and simulation-laboratory contexts, Journal of Research in Science Teaching, 48(1), 71-93.DOI: 10.1002/ tea.20386.

KOVAC, J. (1999), Student Active Learning Methods in General Chemistry, Journal of Chemical Education, 76 (1), 120-124. DOI: 10.1021/ed076p120
MAZUR, E. (1997), Peer instruction: A user's Manual, Prentice Hall Incorporation, New Jersey, EE.UU. MINISTERIO DE EDUCACIÓN, CULTURA Y DEPORTE, Secretaría General de Universidades, Subdirección General de Coordinación y Seguimiento Universitario, (2012), Datos y cifras del sistema universitario espanol, curso 2012-2013. Editado por la Secretaría General Técnica, Subdirección General de Documentación y Publicaciones. Edición 2012. Madrid. España

STERNBERG, R.J., JARVIN, L., GRIGORENKO,E.L.(2009), Teaching for Wisdom, Inteligence, Creativity and Success, Corwin, EE.UU.

TORTOSA MORENO, M. Y GORCHS ALTARRIBA, R. (2006), Estrategias para mejorar el aprendizaje en las prácticas de Química: contextualización y aprendizaje cooperativo, en Comunicación presentada en $4^{\circ}$ Congreso Internacional sobre Docencia Universitaria e Innovación (IV CIDUI), Barcelona.

TORTOSA MORENO, M. Y GORCHS ALTARRIBA R. (2011), Análisis de las prácticas de Química de futuros ingenieros: propuestas de mejora efectivas en el marco del Espacio Europeo de Educación Superior, Revista Electrónica de Enseñanza de las Ciencias, 10 (3), 531-549.

WINDSCHITL, M. (2000), Supporting the development of science inquiry skills with special classes of software, Educational Technology Research 
and Development, 48 (2), 81-95. DOI: 10.1007/BF02313402.

WINN, W., STAHR, F., SARASON, C., FRULAND, R., OPPENHEIMER, P., Y LEE, Y.-L. (2006), Learning oceanography from a computer simulation compared with direct experience at sea, Journal of Research in Science Teaching, 43 (1), 25-42. DOI: 10.1002/tea.20097.

WOELK, K. (2008), Optimizing the Use of Personal Response Devices (Clickers) in Large-Enrollment
Introductory Courses, Journal of Chemical Education, 85 (10), 14001405. DOI: 10.1021/ed085p1400.

\section{ZACHARIAS C. Z. Y CONSTANTINOS} P. C., (2008), Comparing the influence of physical and virtual manipulatives in the context of the Physics by Inquiry curriculum: The case of undergraduate students' conceptual understanding of heat and temperature, American Journal of Physics, 76, 425-430. DOI: 10.1119/1.2885059. 


\section{ANEXO 1: GUÍA DE LABORATORIO PARA LA PRÁCTICA 7}

\section{Heat of neutralization}

Student name:

Go to http://group.chem.iastate.edu/Greenbowe/sections/projectfolder/animationsindex.htm

And then to the third simulation in Thermochemistry section.

You can also go directly to:

http://group.chem.iastate.edu/Greenbowe/sections/projectfolder/animationsindex.htm

Become familiar with different acids and bases.

\section{Purpose.}

Estimate the heat of neutralization for the reaction of $\mathrm{NaOH}$ and $\mathrm{HCl}$.

\section{Introduction:}

Consider the neutralization of hydrochloric acid ( $\mathrm{HCl})$ and sodium hydroxide $(\mathrm{NaOH})$ solutions as shown in the following equation:

$\mathrm{HNO}_{3}(\mathrm{aq})+\mathrm{NaOH}(\mathrm{aq})$ à $\mathrm{H}_{2} \mathrm{O}(\mathrm{l})+\mathrm{Na} \mathrm{NO}_{3}(\mathrm{aq})(\mathrm{a})$

Neutralization reactions are exothermic, that is, energy is released in the form of heat. Heat of neutralization ( $\Delta$ Hneut) is tabulated per mole of reacting substance, and is expressed either in $\mathrm{KJ} / \mathrm{mol}$ or in $\mathrm{Kcal} / \mathrm{mol}$.

Use the simulation with this example and make sure you know how to execute the different measurements and why.

Example: Determine the $\Delta \mathrm{Hneut}$ acid of nitric acid, $\mathrm{HNO}_{3}$, with $\mathrm{NaOH}$ solution. For it, mix $50 \mathrm{~mL}$ of $1.0 \mathrm{M} \mathrm{HNO}_{3}$ and $50 \mathrm{~mL}$ of $1.0 \mathrm{M} \mathrm{NaOH}$. Assume that the temperature of both solutions before mixing them is the same and equal to $23.1^{\circ} \mathrm{C}$, and that you measure the temperature while mixing and after and the maximum recorded is $29.4^{\circ} \mathrm{C}$. The heat capacity of the reaction mixture is $3.89 \mathrm{~J} / \mathrm{g}-{ }^{\circ} \mathrm{C}$ and the density of the solutions is $1.04 \mathrm{~g} / \mathrm{mL}$. 
Solution: Use the equation (heat released, $J)=($ total volume of the solution, $\mathrm{mL}$ ) $\mathrm{x}($ density of the solution, $\mathrm{g} / \mathrm{mL}) \mathrm{x}$ ( specific heat capacity of solution, $\left.\mathrm{J} / \mathrm{g}-{ }^{\circ} \mathrm{C}\right) \mathrm{x}(\mathrm{Tfinal}$ - Tinitial, ${ }^{\circ} \mathrm{C}$ )

$\Delta$ Hneut $=$ Vtotalxdsolxcsolx $\Delta \mathrm{T}(1)$

$\Delta$ Hneut $=(100 \mathrm{~mL}) \times(1.04 \mathrm{~g} / \mathrm{mL}) \times\left(3.89 \mathrm{~J} / \mathrm{g}-{ }^{\circ} \mathrm{C}\right) \times(29.4-23.1)^{\circ} \mathrm{C}=2.57 \times 10^{3} \mathrm{~J}$

From eq (a), Number of moles $($ acid $)=$ Number of moles $($ base $)=V($ acid/base $)(L)$ x Molarity (2)

Number of moles $=50 \times 10^{-3} \mathrm{~L} \times 1 \mathrm{M}=5 \times 10^{-2}$ moles

To get heat per mole, divide the result of (1) by the result of (2).

$\Delta$ Hneut $/ \mathrm{mole}=51.5 \times 10^{3} \mathrm{~J} / \mathrm{mol}$

\section{Procedure:}

1. Use $1 \mathrm{M} \mathrm{HCl}$ and $1 \mathrm{M} \mathrm{NaOH}$.

2. Chose $20 \mathrm{~mL}$ of $\mathrm{HCl}$ and 20 of $\mathrm{NaOH}$.

3. Record the initial temperature.

4. Mix the solutions and record the evolution of temperature with time for 8 minutes each 30 s until minute 4 and each minute after.

5. Plot the graph on an Excel sheet and extrapolate maximum temperature reached. Record this value.

6. Change volumes by $5 \mathrm{~mL}$ up to $50 \mathrm{~mL}$ and repeat steps 3 to 5 for each.

7. Calculate the $\Delta \mathrm{Hneut} / \mathrm{mole}$ for each chosen volume as in the previous example.

8. With the collection of calculated heats, obtain the mean and standard deviation by using Excel.

9. Compare the mean value with the tabulated in the handbook of chemical physics in the lab.

Sign of student. 


\section{ANEXO 2: GUÍA DE LABORATORIO PARA LA PRÁCTICA 8.}

Chemistry laboratory

Reactions: Combustion.

Student name:

\section{A. Introduction.}

When substances take part in a chemical reaction, atoms rearrange to form other substances. The substances that react are called reactants, those obtained are called products, and an arrow ( $($ ) is used to indicate the direction in which the reaction takes place.

Combustion reaction is a kind of reactions used to get energy because heat is one of the products. Reactions in which heat is released are called EXOTHERMIC reactions. Not only fuels are combusted to produce energy in engines, also carbohydrates are combusted in our body to and energy is obtained for our movements and other internal actions. Fats are first stored and only spent when carbohydrates are spent.

Combustion reactions are used to determine with high precision the heat released and therefore to know the most efficient fuel, or the calories contribution in nutrition. The set up needed is very, very expensive and only some research laboratories can afford it.

\section{B. Procedure.}

To perform a combustion reaction follow the next steps:

1. Go to http://www.chem.iastate.edu/group/Greenbowe/sections/projectfolder/ flashfiles/stoichiometry/stoic_excess_oxy.html

2. Click on step 1 and select the hydrocarbon.

3. Write the chemical equation with formulas and put below the names of the reactants and products.

4. Identify in your data sheet reactants and products for each case

5. Predict the coefficients of your balanced equation, and then check with the simulation.

Hydrocarbon 1: 
Hydrocarbon 2:

Hydrocarbon 3:

General hydrocarbon:

6. Look at the products. What do they have in common these four reactions?

7. Why do you think the temperature is measured?

8. Taking into account the two previous questions, what conclusion/conclusions can you reach about combustion reactions?

9. Regarding the setup components, draw it in your data sheet (or copy and paste the image). Could you name them? Do some internet research if needed, or/and ask your instructor if needed.

10. Taking into account the labs performed (how to measure mass and how to measure volume) and the information that you have in this course, could you determine how much of a gas you put in a reaction? Can you measure the volume with any of the introduced volume devices, or the mass with a balance? Argue your answer.

11. Research on the internet/book on how to measure the amount of substance when it is in the gas phase. Ask your instructor if needed (chapter 5 of the text book deals with gases).

12. Click on http://www.chem.iastate.edu/group/Greenbowe/sections/projectfolder/flashfiles/gaslaw/boyles_law_graph.html to clarify this issue.

13. Observations/ conclusions on this laboratory.

Sign of student. 


\section{ANEXO 3: ENCUESTA QUE SE PASA A LOS ESTUDIANTES}

\section{Questions on teaching with simulations:}

Degrees of agreement: 1 (TA: total agreement) 2(A: agreement) 3(N: Neutral) 4 (D: Disagreement) 5 (TD: Total disagreement)

1. Have you found the simulations helpful?

$\begin{array}{lllll}1 & 2 & 3 & 4 & 5\end{array}$

Comments/ Suggestions:

2. Has it contributed to your understanding of these reactions?

$$
\begin{array}{lllll}
1 & 2 & 3 & 4 & 5
\end{array}
$$

Comments/ Suggestions:

3. Do you find simulations more understandable than numerical exercises or questions?

$\begin{array}{lllll}1 & 2 & 3 & 4 & 5\end{array}$

Comments/ Suggestions:

4. Have you properly understood acid-base reactions qualitatively by means of this simulation?

$\begin{array}{lllll}1 & 2 & 3 & 4 & 5\end{array}$

Comments / Suggestions:

5. Have you properly understood acid-base reactions quantitatively by means of this simulation?

$\begin{array}{lllll}1 & 2 & 3 & 4 & 5\end{array}$

Comments / Suggestions: 
6. Have you properly understood qualitatively combustion reactions by means of this simulation?

$\begin{array}{lllll}1 & 2 & 3 & 4 & 5\end{array}$

Comments / Suggestions:

7. Have you properly understood how heat evolution is measured in combustion reaction experiments.

$\begin{array}{lllll}1 & 2 & 3 & 4 & 5\end{array}$

Comments / Suggestions:

General observations:

Advantages and disadvantages of the use of simulation instead of, (or combined with) lectures

Advantages and disadvantages of the use of simulation instead of performing the laboratory experiment. 
